\title{
Assessment of burnout prevention and wellness programs for US-based neurosurgical faculty and residents: a systematic review of the literature
}

\author{
*Laura Berardo, BA, ${ }^{1}$ Christina Gerges, BS, ${ }^{2}$ James Wright, MD, ${ }^{2,3}$ Amber Stout, MLIS, ${ }^{3}$ \\ Hamid Shah, MD, ${ }^{4}$ Alexander Papanastassiou, MD, ${ }^{5}$ and Kristopher Kimmell, MD, ${ }^{6}$ in affiliation \\ with the Council of State Neurosurgical Societies (CSNS)
}

\begin{abstract}
${ }^{1}$ School of Medicine, University of Texas at San Antonio, Texas; ${ }^{2}$ School of Medicine, Case Western Reserve University, Cleveland, Ohio; ${ }^{3}$ Department of Neurosurgery, University Hospitals Cleveland Medical Center, Cleveland, Ohio; ${ }^{4}$ Department of Neurosurgery, Vanderbilt University Medical Center, Nashville, Tennessee; ${ }^{5}$ Department of Neurosurgery, UT Health San Antonio, Texas; and ${ }^{6}$ Department of Neurosurgery, Rochester Regional Health and University of Rochester Medical Center, Rochester, New York
\end{abstract}

OBJECTIVE Neurosurgeon burnout is a serious and prevalent issue that has been shown to impact professionalism, physician health, and patient outcomes. Interventions targeting physician burnout primarily focus on improving physician wellness. Many academic neurosurgery programs have established wellness curricula to combat burnout and improve wellness. No official recommendations exist for establishing a wellness program that effectively targets sources of burnout. The aim of this review was to examine measures of burnout and report objective results of wellness interventions for neurosurgical faculty and residents.

METHODS Two systematic literature reviews were performed in parallel, in accordance with PRISMA 2009 guidelines. Following removal of duplicates, a query of PubMed/MEDLINE, Scopus, Ovid, Cochrane, and EMBASE databases yielded 134 resident-related articles and 208 faculty-related articles for abstract screening. After abstract screening, 17 articles with a primary focus of resident wellness and 10 with a focus on faculty wellness met criteria for full-text screening. Of the total 27 screened articles, 9 ( 6 resident, 2 faculty, 1 both resident and faculty) met criteria and were included in the final analysis. Article quality was assessed using the Joanna Briggs Institute critical appraisal tools for cohort studies.

RESULTS Included studies reported burnout rates for neurosurgery residents of $30 \%-67 \%$. Work-life imbalance, imbalance of duties, inadequate operative exposure, and hostile faculty were contributors to burnout. The 2 included studies reported burnout rates for neurosurgery faculty members of $27 \%$ and $56.7 \%$. Psychosocial stressors, relational stressors, and financial uncertainty were generally associated with increased feelings of burnout. Of the 4 studies reporting on outcomes of wellness initiatives included in this review, 3 reported a positive impact of the wellness interventions and 1 study reported no significant improvement after implementing a wellness initiative.

CONCLUSIONS Burnout among neurosurgical faculty and residents is prevalent and permeates the daily lives of neurosurgeons, negatively affecting patient outcomes, career satisfaction, and quality of life. Many neurosurgery programs have instituted wellness programs to combat burnout, but few have published evidence of improvement after implementation. While studies have shown that residents and faculty recognize the importance of wellness and look favorably on such initiatives, very few studies have reported objective outcomes.

https://thejns.org/doi/abs/10.3171/2020.6.JNS201531

KEYWORDS physician wellness; physician wellbeing; physician burnout; wellness initiative; job satisfaction; occupational health

ABBREVIATIONS ACS = American College of Surgeons; GAD-7 = Generalized Anxiety Disorder 7-ltem scale; JBI = Joanna Briggs Institute; MBI = Maslach Burnout Inventory; MSPSS = Multidimensional Scale of Perceived Social Support; PCP = primary care physician; PGY = postgraduate year; $P H Q-8=$ Personal Health Questionnaire depression scale.

SUBMITTED May 2, 2020. ACCEPTED June 9, 2020.

INCLUDE WHEN CITING Published online October 30, 2020; DOI: 10.3171/2020.6.JNS201531.

${ }^{*}$ L.B. and C.G. contributed equally to this work. 
$\mathrm{P}$ HYSICIAN burnout is a serious and prevalent issue that has been shown to impact professionalism, physician health, and patient outcomes. ${ }^{1}$ Manifestations of burnout include emotional exhaustion, depersonalization or cynicism, and feelings of low accomplishment. ${ }^{2}$ It has been attributed to personal dissatisfaction, cynicism, and "moral injury," a term that has been used to describe the emotional response to ego-dystonic events. ${ }^{3-5}$ Burnout is also associated with higher rates of depression and anxiety, substance use disorders, interpersonal relationship difficulties, and self-reported substandard patient care. ${ }^{2}$ Numerous studies have estimated the prevalence of physician burnout, with rates ranging from $40 \%-70 \%$ across all specialties. ${ }^{2,6-11}$ Due to its prevalence and detrimental effects on the health of physicians and patients, physician burnout has become a targeted area of much-needed improvement.

Interventions targeting physician burnout primarily focus on improving physician wellness, which is a broad, vague, and multifaceted element of physician health. Wellness may include physical, emotional, and mental wellbeing, work-life balance, quality of life, coping mechanisms, achieving success, and thriving professionally. ${ }^{12}$ Current interventions address multiple dimensions of wellness, targeting sources of burnout, and bolstering resistance to burnout. It is worth noting, however, that it has been theorized that these wellness interventions address factors that contribute to burnout, rather than addressing the underlying cause. ${ }^{4}$ These interventions aim to improve physician resilience to mitigate burnout, as combating the personal, professional, and emotional demands that directly cause burnout is a significant undertaking.

The field of neurological surgery is not spared from burnout. A recently published study reported on the results of an international, social media-broadcasted 16-question survey that aimed to assess neurosurgery resident risk of burnout. ${ }^{13}$ They reported that $20.2 \%$ of respondents were at risk of burnout, with North Americans (Canadians and Americans) experiencing the lowest risk (11.2\%) and $\mathrm{Eu}-$ ropeans experiencing the highest (26.9\%). In response to the pervasiveness of burnout, many programs have established wellness curricula to combat burnout and improve physician wellness. One such study by Wolfe et al. compared wellness initiatives by several residency programs. ${ }^{14}$ Wellness protocol components ranged from gym classes, to Fitbit device tracking, to wellness lectures and class social events, tailored to resident needs. ${ }^{14}$ The focus of each wellness initiative varied, but every program emphasized the utility of wellness programs and sought-after positive feedback from the participants. Three initiatives included faculty members, although participation in the program varied.

No official recommendations exist for establishing a residency wellness program that effectively targets sources of burnout. While most wellness programs track effectiveness through resident surveys, there remains a need for more objective data to determine program effectiveness at combating burnout. The aim of this review was to examine measures of burnout and to report objective results of wellness interventions for neurosurgical faculty and residents.

\section{Methods}

A systematic literature review was performed in accordance with PRISMA 2009 guidelines. The PubMed/MEDLINE, Scopus, Ovid, Cochrane, and EMBASE databases were queried for the following terms: ("neurosurgery" OR "neurosurgery") AND ("internship and residency" OR ("internship" AND "residency") OR "internship and residency" OR "residency") AND ("health" OR "health" OR "wellness"). The results of the search were uploaded onto Rayyan Qatar Computing Research Institute Software. ${ }^{15}$

In parallel, PubMed/MEDLINE, Scopus, Ovid, Cochrane, and EMBASE were queried for the following terms: ("neurosurgery" OR "neurosurgical procedures" OR "neurosurgeons" OR "neurosurg" OR "neurological surg") AND ("faculty, medical" OR "hospitalists" OR "faculty" OR "attending") AND ("burnout, psychological" OR "burnout" OR "burn-out" OR "burnt-out" OR "burned out" OR "job satisfaction" OR "career satisfaction" OR "occupational stress" OR "work-related stress" OR "occupational health" OR "work-life balance" OR "compassion fatigue" OR "mental health" OR "workplace wellbeing" OR "wellness program").

Following removal of duplicates, 134 resident-related articles and 208 faculty-related articles met criteria for abstract screening. Abstracts were reviewed by two independent reviewers (L.B. and J.W.) for determination of full-text analysis. All conflicts were decided by a third arbiter (C.G.). Inclusion criteria for abstract screening included original research articles, programs in the US only, the articles must address neurosurgical resident or faculty wellness, and the articles must report objective measures of outcomes. Exclusion criteria included case reports and series of less than 10 participants, articles published in non-English languages, and articles reporting outcomes for programs outside the US. Objective data of interest included measurements of physical and mental health parameters. Primary outcome measures include any reported objective data as well as lowered burnout rates and self-reported measures of resident and faculty wellness.

After abstract screening, 17 articles with a primary focus of resident wellness and 10 with a focus on faculty wellness met criteria for full-text screening. Article eligibility was decided based on inclusion criteria as follows: the article specifically addressed causes of neurosurgeon burnout and its relation to wellness, specific measures taken to improve neurosurgeon wellness, or an analysis of a wellness initiative. Of the 27 total screened articles, 9 met criteria and were included in the final analysis (Fig. 1). Article quality was assessed using the Joanna Briggs Institute (JBI) critical appraisal tool for cohort studies (Supplemental Table 1). ${ }^{16}$ Using an 11-question checklist that is completed by two reviewers and an arbitrator, when necessary, this tool allows for critical assessment of the methodological quality of each included article.

\section{Results}

Of the 9 studies that were included in the final analysis, 6 reported data exclusively on resident wellness and 2 exclusively on faculty wellness. One study reported data for 
Review of Resident Wellness/Burnout Literature
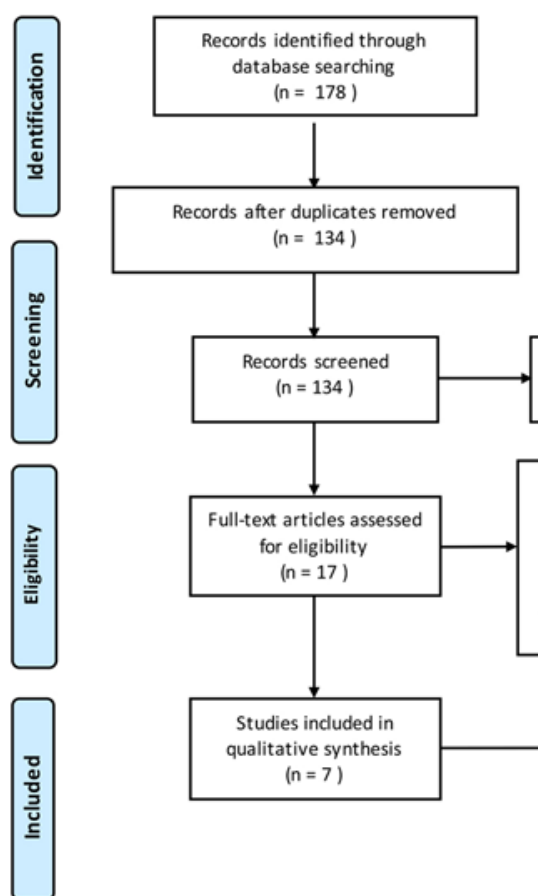

Studies included in qualitative synthesis $(n=7)$

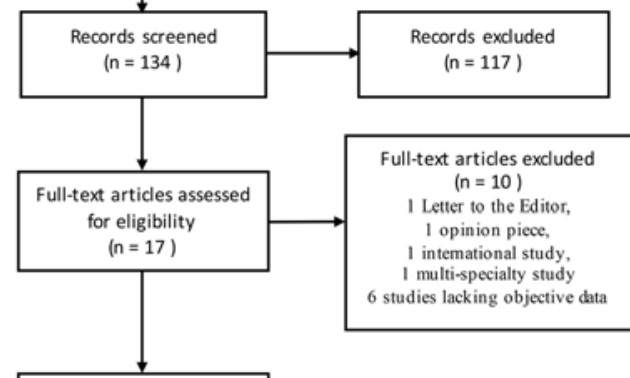

Review of Faculty Wellness/Burnout Literature
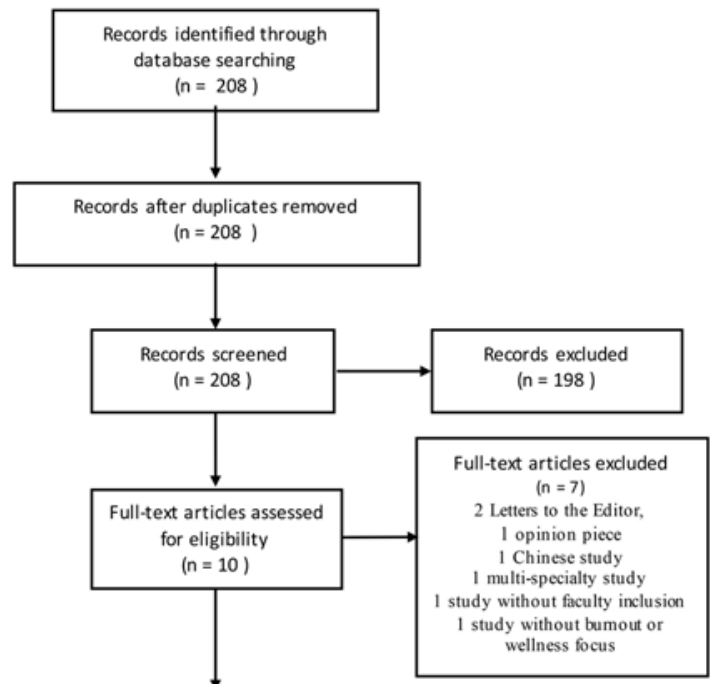

Studies included in qualitative synthesis $(n=3)$

FIG. 1. Two systematic literature reviews, performed in accordance with 2009 PRISMA guidelines, were conducted in parallel and later combined. Figure is available in color online only.

both faculty and resident wellness. Study data are outlined in Table 1

\section{Resident Burnout}

Included studies reported burnout rates for neurosurgery residents of $30 \%-67 \%$ (Table 2) ${ }^{17-20}$ Of the articles that provided demographic data, all postgraduate years
(PGYs) were surveyed equally and all regions of the US were included. ${ }^{17,18,20}$ Work-life imbalance, imbalance of duties, inadequate operative exposure, and hostile faculty were contributors to burnout. ${ }^{17,20}$ End of rotation, end of PGY, increased sleep, outdoor activities, and intraprogram social events contributed to lower feelings of burnout among resident neurosurgeons in 1 study. ${ }^{17}$ Meaningful

TABLE 1. Summary of studies reporting on burnout or wellness of neurosurgeons

\begin{tabular}{lcccc}
\hline \multicolumn{1}{c}{ Authors \& Year } & Single or Multiinstitution & Intervention & Study Population & QoE Score (JBI) \\
\hline Klimo et al., $2013^{9}$ & Multiinstitution & Online survey & Faculty & $8 / 11$ \\
\hline McAbee et al., $2015^{21}$ & Multiinstitution & Online survey & Faculty & $8 / 11$ \\
\hline Spiotta et al., $2016^{22}$ & Single institution & Wellness initiative & Residents \& faculty & $8 / 11$ \\
\hline Buchholz et al., $2018^{24}$ & Single institution & Online survey & Residents & $9 / 11$ \\
\hline Attenello et al., $2018^{20}$ & Multiinstitution & Online survey & Residents & $9 / 11$ \\
\hline Ares et al., $2019^{19}$ & Single institution & Wellness initiative & Residents & $8 / 11$ \\
\hline Spiotta et al., $2019^{23}$ & Single institution & Wellness initiative & Residents & $9 / 11$ \\
\hline Shakir et al., $2020^{18}$ & Multiinstitution & Online survey & Residents & $8 / 11$ \\
\hline Tang et al., $2020^{17}$ & Single institution & Online survey & Residents & $9 / 11$ \\
\hline
\end{tabular}

QoE = quality of evidence. 


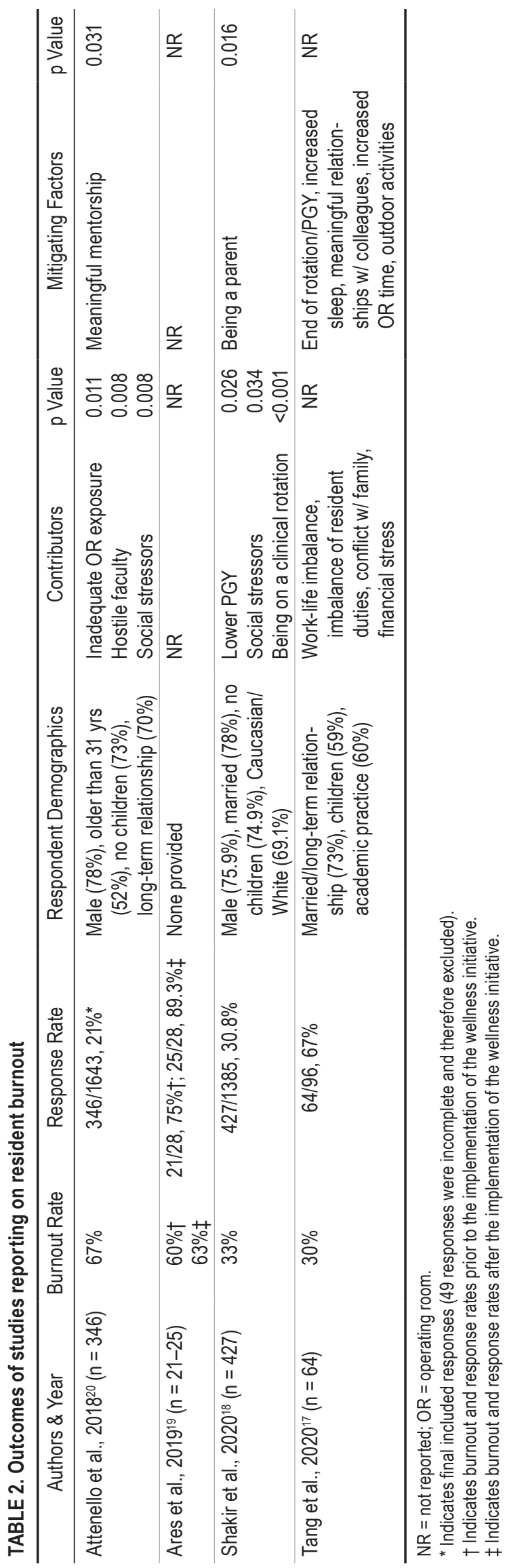

mentorship and relationships with colleagues were found to be protective against burnout. ${ }^{17,20}$ Another study reported that high "grit" (defined as sustained effort over an extended time; $p=0.006)$, being married $(p=0.025)$, and having fewer social/personal stressors $(\mathrm{p}=0.003)$ were protective factors against the development of burnout. ${ }^{18}$

\section{Faculty Burnout}

Included studies reported burnout rates for neurosurgery faculty members of $27 \%$ and $56.7 \%$ (Table 3 ). ${ }^{10,21}$ One study surveyed all US members of the American Association of Neurological Surgeons (AANS), while the other did not report geographical data. ${ }^{21}$ Psychosocial stressors, relational stressors, and financial uncertainty were generally associated with increased feelings of burnout. ${ }^{10,21}$ Other factors such as administrative entanglements, accusations of malpractice, negative patient interactions, uncertainty about future earnings, and dissatisfaction with work-life balance impacted neurosurgeons' careers and influenced burnout. Protective factors included intellectual stimulation, academic affiliation, having children, and having a favorable work-life balance. Importantly, burnout increases career dissatisfaction by almost sixfold (odds ratio 1.7). ${ }^{21}$

In 2008, the American College of Surgeons (ACS) commissioned a study through their Committee on Physician Health and Competency. ${ }^{10}$ This survey included nearly 8000 respondents from multiple surgical disciplines. ${ }^{10}$ The results showed that $71 \%$ of surgeons were satisfied with their careers, but $40 \%$ experienced burnout, $49 \%$ would not recommend surgery as a career, and only $36 \%$ felt they had an appropriate work-life balance..$^{10}$ Comparatively, Klimo et al. found that a majority of neurosurgeons (73\%) were satisfied with their career, some (27\%) reported lower burnout rates, and most (88\%) would choose neurosurgery again. ${ }^{9}$

\section{Wellness Initiatives}

Of the 4 studies reporting on outcomes of wellness initiatives included in this review, 3 reported a positive impact of the wellness interventions, ${ }^{22-24}$ whereas 1 study reported no significant improvement after implementing a wellness initiative (Table 4). ${ }^{19}$

The 3 articles reporting significant outcomes reported on the same wellness initiative. Each paper reported on unique outcome measures at different time periods. The wellness initiative referred to as "La Sierra" included 9 residents and 6 faculty members initially. ${ }^{22}$ However, after 3 years, the study population was limited to 8 residents..$^{23,24}$ They collected baseline information regarding physical health, mental health, and quality of life and implemented an initiative to improve those domains. ${ }^{22}$ The initiative included weekly lectures on wellness topics such as exercise, diet, and mental health practices, weekly team-based exercise sessions aided by physical trainers, and provision of healthy food options at department conferences. ${ }^{25}$ Each participant was given a Fitbit device, which tracked sleep, activity levels, and metabolic rate. Exercise, dietary, and sleep goals were used to promote progress and were tracked through surveys. ${ }^{25}$ Results of the intervention were tracked for years after its implementation. 
TABLE 3. Outcomes of studies reporting on faculty burnout

\begin{tabular}{|c|c|c|c|c|c|c|c|}
\hline Authors \& Year & $\begin{array}{l}\text { Burnout } \\
\text { Rate }\end{array}$ & $\begin{array}{l}\text { Response } \\
\text { Rate }(n, \%)\end{array}$ & Respondent Demographics & Contributors & $p$ Value & Mitigating Factors & $p$ Value \\
\hline $\begin{array}{l}\text { Klimo et al., } \\
2013^{9}(n=85)\end{array}$ & $27 \%$ & $85 / 169,50.3 \%$ & $\begin{array}{l}\text { Male }(96 \%) \text {, White/ } \\
\text { Caucasian (94\%), children } \\
(91 \%) \text {, academic practice } \\
(56 \%), 5-10 \text { yrs in } \\
\text { practice }(29.4 \%)\end{array}$ & $\begin{array}{l}\text { Administrative entangle- } \\
\text { ments, negative patient } \\
\text { interactions, insuf- } \\
\text { ficient time control }\end{array}$ & NR & $\begin{array}{l}\text { Treating patients, intel- } \\
\text { lectual stimulation, } \\
\text { prestige, colleagues, } \\
\text { teaching, research }\end{array}$ & NR \\
\hline $\begin{array}{l}\text { McAbee et al., } \\
2015^{21}(n=750)\end{array}$ & $56.7 \%$ & $783 / 3247,24.1 \%$ & $\begin{array}{l}\text { Male }(87.3 \%) \text {, older than } 40 \\
\text { yrs }(88 \%), \text { married/ long- } \\
\text { term relationship ( } 91.1 \%) \text {, } \\
\text { academic practice } \\
(25.5 \%)\end{array}$ & $\begin{array}{l}\text { Malpractice accusation } \\
\text { Uncertainty about future } \\
\text { earnings }\end{array}$ & $\begin{array}{r}0.004 \\
<0.001\end{array}$ & $\begin{array}{l}\text { Academic affiliation } \\
\text { Work-life balance } \\
\text { Challenged at work } \\
\text { Understanding spouse } \\
\text { Adequate time for per- } \\
\quad \text { sonal development } \\
\text { Having children }\end{array}$ & $\begin{array}{c}0.03 \\
<0.001 \\
0.02 \\
0.001 \\
0.005 \\
0.01\end{array}$ \\
\hline
\end{tabular}

TABLE 4. Outcomes of studies reporting on wellness initiatives

\begin{tabular}{|c|c|c|c|c|}
\hline Authors \& Year & Outcome Measure & Preintervention & Postintervention & $p$ Value \\
\hline \multirow{8}{*}{ Spiotta et al., $2016^{22}(n=15)$} & Elevated systolic BP & $60 \%$ & NR & NR \\
\hline & Elevated diastolic BP & $47 \%$ & & \\
\hline & Elevated serum LDL & $53 \%$ & & \\
\hline & Elevated body weight & $69 \%$ & & \\
\hline & PHQ-8 & 5.0 (8\% abnormal) & & \\
\hline & GAD-7 & 4.6 (8\% abnormal) & & \\
\hline & Quality of Life Scale & 77.7 (77\% abnormal) & & \\
\hline & Epworth Sleepiness Scale & 8.0 (31\% abnormal) & & \\
\hline \multirow{11}{*}{$\begin{array}{l}\text { Ares et al., } 2019^{19} \text { (preintervention, } n=21 \text {; } \\
\text { postintervention, } n=25 \text { ) }\end{array}$} & Self-care & & & \\
\hline & PCP visit (last year) & $33 \%$ & $32 \%$ & 0.92 \\
\hline & Dentist visit (last year) & $71 \%$ & $44 \%$ & 0.06 \\
\hline & $\mathrm{MBI}$ & & & \\
\hline & Emotional exhaustion & 15.9 & 16 & 0.92 \\
\hline & Depersonalization & 7.6 & 7.4 & 0.83 \\
\hline & Personal accomplishment & 6.5 & 5.8 & 0.59 \\
\hline & MSPSS & & & \\
\hline & Significant other & 6.4 & 6.2 & 0.64 \\
\hline & Friends & 6.1 & 6.4 & 0.20 \\
\hline & Family & 6.6 & 6.4 & 0.41 \\
\hline \multirow{5}{*}{ Spiotta et al., $2019^{23}(n=8)$} & PHQ-8 & 4.1 & 2.8 & $>0.05$ \\
\hline & GAD-7 & 4.0 & 2.1 & 0.039 \\
\hline & Quality of Life Scale & 82.4 & 95.4 & 0.007 \\
\hline & Epworth Sleepiness Scale & 8.3 & 5.7 & 0.019 \\
\hline & \multicolumn{2}{|c|}{ Survey Question } & Agreement & p Value \\
\hline \multirow{6}{*}{ Buchholz et al., $2018^{24}(n=8)$} & \multicolumn{4}{|l|}{ "Wellness Initiative was... } \\
\hline & \multicolumn{2}{|l|}{ very important for residents." } & $100 \%$ & NR \\
\hline & \multicolumn{2}{|l|}{ largely positive for department." } & $100 \%$ & \\
\hline & \multicolumn{2}{|c|}{ beneficial to both professional and personal life." } & $87.5 \%$ & \\
\hline & \multicolumn{2}{|c|}{ "I am frequently concerned about my health." } & $87.5 \%$ & \\
\hline & \multicolumn{2}{|c|}{ "I feel more prepared to perform my work and patient care duties." } & $62.5 \%$ & \\
\hline
\end{tabular}

$\mathrm{BP}=$ blood pressure; $\mathrm{LDL}=$ low-density lipoprotein 
The baseline physical assessments found abnormalities in $80 \%$ of participants, most commonly hypertension and hypercholesterolemia. ${ }^{22}$ Body composition reported an average body fat percentage of $21.5 \%$, and BMI analysis revealed that $47 \%$ of participants were overweight and $13 \%$ obese. ${ }^{22}$ After only 4 months of the initiative, the participants experienced an average weight loss of 3.6 pounds ( $1 \%$ body fat). ${ }^{22}$

Sleepiness, which was assessed with the Epworth Sleepiness Scale, was found to be significantly improved postintervention (preintervention score $=8.3$, postintervention score $=5.7, p=0.019) .{ }^{23}$ Mental health, which was assessed with the Generalized Anxiety Disorder 7-Item scale (GAD-7), was found to be significantly improved postintervention (preintervention score $=4$, postintervention score $=2.1, p=0.039) \cdot{ }^{23}$ Quality of life, as measured by the Quality of Life Scale, was found to be significantly improved after the intervention (preintervention score = 82.4, postintervention score $=95.4, \mathrm{p}=0.007$ ). . Depression, as assessed by the Personal Health Questionnaire depression scale (PHQ-8), was unchanged in this study cohort. ${ }^{23}$

At that time, the initiative was well received, with $64 \%$ of participants responding that the program improved health and mental wellbeing and $91 \%$ of participants reporting improved team building and camaraderie. ${ }^{23}$ None of the participants reported interference with clinical duties. ${ }^{25}$ In a later survey, residents had generally positive views toward the program, recognized the importance of a successful wellness initiative, reported positive promotion of team building and camaraderie, and reported that the program did not negatively affect physician-patient care responsibilities. ${ }^{25}$

A study by Buchholz et al. reported resident responses to the implementation of the La Sierra wellness initiative 3 years after implementation. All of the participants (100\%) believed that the wellness initiative was very important for residents and had a largely positive impact on the department. ${ }^{24}$ Survey responses showed that a strong majority $(87.5 \%)$ felt an improvement in their mental wellbeing and believed the program was beneficial to personal and professional life. ${ }^{24} \mathrm{~A}$ majority of respondents (62.5\%) felt more prepared to perform patient care and felt a positive impact on sleep quality. ${ }^{24}$ Interestingly, most residents did not report an impact on perceived overall self-performance. ${ }^{24}$

A unique initiative described by Ares et al. ${ }^{19}$ aimed to improve physical health, mental health, spiritual life, and interpersonal relationships to improve work-life balance and combat burnout. The initiative included bimonthly wellness lectures, free access to the hospital gym for all residents, group gym visits, and renewed focus on mentorship. ${ }^{19}$ Survey results showed that $52 \%$ of residents believed free gym access was a major contributor to their physical health and $60 \%$ thought lectures were very or mildly helpful. ${ }^{19}$ Wellness was tracked by self-care measures, such as visits to their dentist or primary care physician (PCP) within the past year, the Maslach Burnout Inventory (MBI), and the Multidimensional Scale of Perceived Social Support (MSPSS). Overall, no statistical difference was found between pre- and postintervention groups for any of the measures examined..$^{19}$

\section{Discussion \\ Burnout in Neurosurgery}

Physician burnout is a prevalent problem in the medical community. Neurosurgeons are as affected by burnout as physicians in other specialties, with cited burnout rates ranging from $27 \%$ to $67 \% .^{10,17,20,21,26}$ Comparatively, recorded burnout rates of other specialties range from $9 \%$ (ophthalmology chairs) to $45 \%$ (clinical oncologists). ${ }^{21} \mathrm{~A}$ 2009 study surveying 7905 American surgeons found an overall burnout rate of approximately $40 \% .{ }^{21}$ Studies reporting higher rates of burnout in neurosurgery attribute this to higher depersonalization scores, long hours, and intense workload. ${ }^{17,21}$ Scores for emotional exhaustion are also high, likely due to the demanding workload and difficult cases. Additionally, studies reporting lower rates of burnout in neurosurgery attribute the phenomenon to higher rates of personal accomplishment. ${ }^{18}$

A significant factor associated with burnout is poor work-life balance. Long hours and difficult work make desired work-life balance challenging to achieve. Perception of good work-life balance alone contributes to lower rates of burnout. Conversely, poor work-life imbalance is independently associated with a higher probability of experiencing burnout. Additionally, social support and lack of socioeconomic stressors protect against burnout. These factors may bolster protection from emotional exhaustion by providing a support system and community. Stressors, both financial and personal, contribute to workload stressors and facilitate emotional exhaustion and burnout. These characteristics have been documented by multiple studies, demonstrating that multiple components affecting wellness contribute to burnout. .0,15, $17,20,21^{2}$

There are disparate rates of burnout reported in these studies. Although most included studies utilized the validated MBI, ${ }^{27}$ this tool was used in varying ways. Some studies utilized the full 22-question survey, ,20,21 while others used an abridged 9-question survey. ${ }^{18}$ Tang et al. ${ }^{17}$ utilized a single-item burnout measure that has been shown to correlate with the MBI. ${ }^{28}$ To ensure standard measurement of burnout, the MBI should be used. Use of the abridged version is acceptable, given rates of survey fatigue in physicians and physician trainees. ${ }^{29}$

A second cause of burnout rate discrepancies may arise due to differing sample populations. Klimo et al. ${ }^{9}$ surveyed academic neurosurgeons, but their comparison article, which reported higher burnout values (38.5 vs $27 \%$ ), surveyed mostly private practitioners. McAbee et al. ${ }^{21}$ noted that their pilot study was primarily academic neurosurgeons and found that the burnout rate was lower compared to peers in private practice in their final study. Their survey data demonstrated that $47.7 \%$ of academics were burned out and had less call and fewer overall hours compared to nonacademics, while $62.8 \%$ of nonacademic neurosurgeons were burned out. ${ }^{21}$ These differences may be a result of differing call and work hours, a difference in work environment, or, most likely, a combination of factors. However, it is important to note this difference, which may necessitate comparison studies.

Other considerations for differing reported rates of neurosurgery include selection bias and survey burnout. Physicians who are suffering from burnout or are dissatis- 
fied with their careers may be less willing to respond to surveys. Additionally, many physicians suffer from "survey burnout," as they are inundated with frequent requests for survey responses..$^{29}$ Physicians may be less likely to respond as a result or may answer less accurately or incompletely if the survey is too long. A few studies noted responses that were incomplete..$^{10,21}$

Burnout rates for residents and faculty were similar (30\%-67\% and $27 \%-56.7 \%$, respectively). ${ }^{10,17,20,21}$ The slightly higher rates during residency may be due to the onset of burnout, increasing maximally during PGY-2 and PGY-4 training years. ${ }^{17}$ Multiple studies reported an increase in resident burnout rates during PGY-2. ${ }^{17,20}$ The relative increase in burnout rates during PGY-2 may be attributable to increased clinical responsibility and a more significant role in the care of neurologically ill patients when compared to PGY-1. Resident burnout rates decline throughout the years, as residents near the end of their training, possibly due to increasing confidence in clinical skills and patient care. Additionally, the end of each PGY was regarded as a mitigator of burnout, potentially due to a sense of accomplishment. One author noted that other specialties have similar spikes in burnout rates during the second year of training, and noted that this may be an optimal time for intervention. ${ }^{20}$ Significant trends in burnout rates among faculty members were not reported in the included studies. However, the authors theorize that heightened stress is experienced early during a career. Young neurosurgeons may be susceptible to burnout due to new levels of responsibility, difficulty with establishing a patient base, new roles in research, and new administrative requirements.

Burnout in the field of neurosurgery is unlikely to disappear. However, for both residents and faculty, it is possible to decrease burnout prevalence and severity by improving work-life balance, reducing personal stressors, and improving mental health.

\section{Wellness Initiatives}

While there is an abundance of evidence that wellness is a critical aspect of physician health, there are few published interventions targeting neurosurgery resident wellness, and even fewer programs exist for faculty members. While many residency programs have established wellness initiatives, few have been evaluated for effectiveness. Although the presence of wellness initiatives has been shown to have a largely positive impact on neurosurgery departments, there remains a need to systematically evaluate such programs to ensure positive impact on the psychological and physical wellbeing of neurosurgeons.

This review of the literature reports on two unique wellness initiatives implemented at academic neurosurgery departments. ${ }^{18,22-24}$ The first of the two initiatives, La Sierra, was the basis for 3 of the studies included in this review. These studies showed significant improvement in the psychological wellbeing of their participants through implementation of the wellness initiative. The final study population, consisting of 8 residents of "very diverse" backgrounds, was considered to be representative of resident neurosurgeons. Although part of the initial study population, all faculty members were excluded from the study in the 3 years between publication of baseline data and outcomes of the intervention. ${ }^{23,24}$ As such, generalizability of the results to faculty members is not possible. Furthermore, an inability to retain faculty members as participants may suggest its ineffectiveness as an intervention in that population. Ares et al. published a singleinstitution study that showed no statistical difference in self-care measures, MBI, or MPSS after implementation of a wellness initiative. ${ }^{19}$

In the Ares et al. 2019 study, residents cited a perceived lack of departmental and faculty support for the initiative. Interviewed residents expressed concern that they would be "shamed" by their peers if they were to prioritize their own physical or psychological wellness. However, no such concerns were cited from residents included in the $\mathrm{La} \mathrm{Si}$ erra initiative. It is possible that faculty inclusion at the onset of the initiative decreased resident perception of stigma associated with taking care of oneself before taking care of one's patients. ${ }^{19}$ Furthermore, several studies have shown that faculty support is a significant mitigator of resident burnout. ${ }^{15,20}$ Given this information, faculty inclusion in wellness initiatives may have a positive effect on resident morale and commitment to improving their own wellness. To measure objective wellness data effectively, standardization of wellness program analysis may be beneficial. A proposed standardization approach could track multiple aspects of wellness: mental wellness with the GAD-7, the PHQ-8, and the Epworth Sleepiness Scale; physical health with baseline data such as blood pressure, BMI, and annual laboratory tests; and emotional wellness with the Quality of Life Scale and the MBI. The success of a wellness initiative is contingent on commitment from the targeted population. Including faculty members and residents in wellness programs allows faculty members to benefit from such programs, while ensuring that residents do not experience perceived stigma. It may also be beneficial to involve residents in the creation of initiatives, as the needs of each institution and its residents may vary.

Interestingly, wellness initiatives for neurosurgical faculty are essentially nonexistent, despite the heightened risk of burnout for early career neurosurgeons. Although the La Sierra initiative initially included 6 faculty members, they were ultimately all excluded from the study. Although the authors did not comment on the loss of this study population, it may be extrapolated that the intervention was not of utility to them. Additionally, there are stark differences in mitigating and compounding factors of burnout cited by faculty members and residents (Tables 2 and 3). These factors suggest that different interventions may be necessary to effectively improve wellness in faculty members and residents.

\section{Limitations}

This review of the literature aimed to examine the topics of burnout prevention and wellness initiatives in neurosurgeons. The relative lack of literature reporting exclusively on neurosurgical faculty members posed a challenge in the review process and prompted the combination of two separate literature reviews into one study. While studies exist, they are skewed toward reporting on resident-centric data. Given the paucity of literature regarding wellness 
initiatives among neurosurgery faculty, there are little data available to substantiate the impact of such programs on that population in particular. Furthermore, the failure of available studies to report on differences in burnout rates among faculty members at different points in their careers resulted in an inability to identify the populations at highest risk of burnout and its sequelae.

\section{Conclusions}

Burnout among neurosurgical faculty and residents is prevalent and permeates the daily lives of those at all career stages. Burnout negatively affects patient outcomes, career satisfaction, and quality of life. Studies have demonstrated factors associated with the development of burnout and have examined wellness interventions that may serve to mitigate or prevent burnout development. Many neurosurgical departments have instituted wellness programs or initiatives to combat burnout, but few have published evidence of improvement after implementation. While studies have shown that residents and faculty recognize the importance of wellness and look favorably on such initiatives, the majority of reported results are subjective in nature. The studies outlined in this work provide a good example of objective measures of effective wellness programs and provide data that might allow for tracking of progression or mitigation of burnout among neurosurgical residents and faculty. More objective data would improve existing wellness programs and might allow for implementation of programs that could improve the quality of life for neurosurgeons at all career stages.

\section{References}

1. West CP, Dyrbye LN, Erwin PJ, Shanafelt TD. Interventions to prevent and reduce physician burnout: a systematic review and meta-analysis. Lancet. 2016;388(10057):2272-2281.

2. Lall MD, Gaeta TJ, Chung AS, et al. Assessment of physician well-being, part one: burnout and other negative states. West J Emerg Med. 2019;20(2):278-290.

3. Rosenstein AH. Hospital administration response to physician stress and burnout. Hosp Pract (1995). 2019;47(5): 217-220.

4. Squiers JJ, Lobdell KW, Fann JI, DiMaio JM. Physician burnout: are we treating the symptoms instead of the disease? Ann Thorac Surg. 2017;104(4):1117-1122.

5. Rozario D. Burnout, resilience and moral injury: how the wicked problems of health care defy solutions, yet require innovative strategies in the modern era. Can J Surg. 2019;62(4): E6-E8.

6. Rotenstein LS, Torre M, Ramos MA, et al. Prevalence of burnout among physicians: a systematic review. JAMA. 2018; 320(11):1131-1150.

7. Ripp J, Babyatsky M, Fallar R, et al. The incidence and predictors of job burnout in first-year internal medicine residents: a five-institution study. Acad Med. 2011;86(10): 1304-1310.

8. Gelfand DV, Podnos YD, Carmichael JC, et al. Effect of the 80-hour workweek on resident burnout. Arch Surg. 2004; 139(9):933-940.

9. Klimo P Jr, DeCuypere M, Ragel BT, et al. Career satisfaction and burnout among U.S. neurosurgeons: a feasibility and pilot study. World Neurosurg. 2013;80(5):e59-e68.

10. Shanafelt TD, Balch CM, Bechamps GJ, et al. Burnout and career satisfaction among American surgeons. Ann Surg. 2009;250(3):463-471.
11. Campbell J, Prochazka AV, Yamashita T, Gopal R. Predictors of persistent burnout in internal medicine residents: a prospective cohort study. Acad Med. 2010;85(10):1630-1634.

12. Lall MD, Gaeta TJ, Chung AS, et al. Assessment of physician well-being, part two: beyond burnout. West J Emerg Med. 2019;20(2):291-304.

13. Jean WC, Ironside NT, Felbaum DR, Syed HR. The impact of work-related factors on risk of resident burnout: a global neurosurgery pilot study. World Neurosurg. 2020;138:e345e353.

14. Wolfe SQ, West JL, Hunt MA, et al. A comparison of the existing wellness programs in neurosurgery and institution champion's perspectives. Neurosurgery. 2019;84(5):11491155 .

15. Ouzzani M, Hammady H, Fedorowicz Z, Elmagarmid A. Rayyan-a web and mobile app for systematic reviews. Syst Rev. 2016;5(1):210.

16 Aromataris E, Munn Z, eds. Joanna Briggs Institute Reviewer's Manual. The Joanna Briggs Institute; 2017.

17. Tang OY, Dunn KA, Yoon JS, et al. Neurosurgery resident wellness and recovery from burnout: a 39-year single-institution experience. World Neurosurg. 2020;138:e72-e81.

18. Shakir HJ, Cappuzzo JM, Shallwani H, et al. Relationship of grit and resilience to burnout among U.S. neurosurgery residents. World Neurosurg. 2020;134:e224-e236.

19. Ares WJ, Maroon JC, Jankowitz BT. In pursuit of balance: the UPMC Neurosurgery Wellness Initiative. World Neurosurg. 2019;132:e704-e709.

20. Attenello FJ, Buchanan IA, Wen T, et al. Factors associated with burnout among US neurosurgery residents: a nationwide survey. J Neurosurg. 2018;129(5):1349-1363.

21. McAbee JH, Ragel BT, McCartney S, et al. Factors associated with career satisfaction and burnout among US neurosurgeons: results of a nationwide survey. J Neurosurg. 2015; 123(1):161-173.

22. Spiotta AM, Fargen KM, Denham SL, et al. Incorporation of a physical education and nutrition program into neurosurgery: a proof of concept pilot program. Neurosurgery. 2016; 79(4):613-619.

23. Spiotta AM, Fargen KM, Patel S, et al. Impact of a residencyintegrated wellness program on resident mental health, sleepiness, and quality of life. Neurosurgery. 2019;84(2): 341-346.

24. Buchholz AL, Henderson F Jr, Lowe S, et al. Perspectives from a residency training program following the implementation of a wellness initiative. World Neurosurg. 2018;119: e947-e955.

25. Fargen KM, Spiotta AM, Turner RD, Patel S. Operation La Sierra: a novel wellness initiative for neurological surgery residents. J Grad Med Educ. 2016;8(3):457-458.

26. Pulcrano M, Evans SRT, Sosin M. Quality of life and burnout rates across surgical specialties: a systematic review. JAMA Surg. 2016;151(10):970-978.

27. Maslach C, Jackson SE, Leiter MP, et al. Maslach Burnout Inventory. Consulting Psychologists Press; 1986.

28. Rohland BM, Kruse GR, Rohrer JE. Validation of a singleitem measure of burnout against the Maslach Burnout Inventory among physicians. Stress Health. 2004;20(2): $75-79$.

29. O'Reilly-Shah VN. Factors influencing healthcare provider respondent fatigue answering a globally administered in-app survey. PeerJ. 2017;5:e3785.

\section{Disclosures}

The authors report no conflict of interest concerning the materials or methods used in this study or the findings specified in this paper. 


\section{Author Contributions}

Conception and design: Wright, Shah, Papanastassiou, Kimmell. Acquisition of data: Wright, Berardo, Gerges, Stout. Analysis and interpretation of data: Wright, Berardo, Gerges. Drafting the article: Wright, Berardo, Gerges. Critically revising the article: Wright, Shah, Papanastassiou, Kimmell. Reviewed submitted version of manuscript: Wright. Approved the final version of the manuscript on behalf of all authors: Wright. Study supervision: Wright, Shah, Papanastassiou, Kimmell.

\section{Supplemental Information}

Online-Only Content

Supplemental material is available with the online version of the article.

Supplemental Table 1. https://thejns.org/doi/suppl/10.3171/ 2020.6.JNS201531.

\section{Correspondence}

James Wright: University Hospitals Cleveland Medical Center, Cleveland, OH. jmonroewright@gmail.com. 